Notre Dame Journal of Formal Logic

Volume 46, Number 2, 2005

\title{
A Negation-free Proof of Cantor's Theorem
}

\author{
N. Raja
}

Abstract We construct a novel proof of Cantor's theorem in set theory.

\section{Introduction}

It has been an important endeavor in logic and mathematics to determine whether the proofs of basic theorems can be reformulated without invoking certain kinds of logical primitives. A striking instance of such a reformulation was provided by Yablo's paradox ([1], [2], [3]) which demonstrated that it was possible to construct paradoxical sentences in logic without the need to invoke either direct or indirect self-reference. We carve another such path in this paper by constructing a new proof of Cantor's theorem in set theory without explicitly invoking the negation operation.

Every proof of Cantor's theorem-that for no set there is a function mapping its members onto all its subsets-constructs a subset which is leftover by any onto mapping from any set to its powerset. The traditional diagonalization proof involves an explicit invocation of the negation operation in order to define the leftover subset. Our new proof of Cantor's theorem, though it uses diagonalization at a certain level, constructs the leftover subset without explicitly invoking the negation operation. Further, our proof can also be rewritten in a form which uses negation explicitly.

\section{Yablo's Paradox}

Yablo's paradox ([1], [2], [3]) is a non-self-referential Liar's paradox. Before the formulation of Yablo's paradox, all known paradoxes in logic seemed to require circularity in an unavoidable way. Each of them used either direct self-reference or indirect looplike self-reference. Yablo's paradox demonstrated that self-reference was not a necessary condition for the construction of paradoxical sentences. It can be stated as follows.

Received April 20, 2004; accepted May 21, 2004; printed May 25, 2005

2000 Mathematics Subject Classification: Primary, 03E99; Secondary, 03F99

Keywords: set theory, Yablo's Paradox, proof complexity

(C)2005 University of Notre Dame 
Consider the following infinite sequence of sentences $S_{i}$ where the indices ' $i, j, k$ ' range over natural numbers:

$$
\left(S_{i}\right): \text { For all } j>i, S_{j} \text { is untrue. }
$$

Note that, in the above sequence of statements, each statement quantifies only over statements which occur later in the sequence. Now suppose $S_{k}$ is true for some $k$. Then $S_{k+1}$ is false, and so are all subsequent statements. As all subsequent statements are false, $S_{k+1}$ is true, which is a contradiction. So $S_{k}$ is false for all $k$. Looking at any particular $i$, this in turn means that $S_{i}$ in fact holds, which is a contradiction.

\section{New Proof of Cantor's Theorem}

Theorem 3.1 (Cantor's Theorem) The cardinality of the power set of a set $X$ exceeds the cardinality of $X$, and in particular the continuum is uncountable.

Proof Let $X$ be any set, and $P(X)$ denote the power set of $X$. Assume that it is possible to define a one-to-one mapping $M: X \leftrightarrow P(X)$.

Define $s_{0}, s_{1}, s_{2}, \ldots$ to be a trace, where the first element of the trace is any arbitrary $s_{0} \in X$, and all further elements $s_{j}$, where $j>0$, of the trace are such that $s_{j} \in M\left(s_{j-1}\right)$. Define $t \in X$ to be a simple element, if all possible traces beginning with $t$ terminate. Note that a trace $s_{0}, s_{1}, s_{2}, \ldots, s_{f}$ terminates at $s_{f}$ if $M\left(s_{f}\right)$ is the empty set. Define $N=\{t \in X \mid t$ is a simple element $\}$.

The set $N$, which is a subset of $X$, cannot lie in the range of $M$. Suppose there exists an $n \in X$ such that $M(n)=N$, then $n$ should be a simple element since all traces beginning with element $n$ also terminate. Thus $n \in N$, but then $n$ is no longer a simple element, since not all traces beginning with $n$ are terminating traces (e.g., " $\mathrm{n}, \mathrm{n}, \mathrm{n}, \ldots$ " is one such nonterminating trace). Thus the set $N$ is out of the range of mapping $M$.

In the above novel proof of Cantor's theorem, the construction of the set $N$ does not require explicit negation. This is unlike the standard diagonalization proof which invokes the operation of negation in order to construct the leftover subset. Of course, one could say the same of the usual diagonal argument showing that the reals are uncountable, because the process of swapping $0 \mathrm{~s}$ and $1 \mathrm{~s}$ in the binary expansion of a real number need not be thought of as negation. Also, it is possible to rewrite the above proof in a slightly different way and bring out negation explicitly. This can be done by changing the definition of a simple element as one whose traces do not continue indefinitely.

\section{References}

[1] Yablo, S., "Truth and reflection," Journal of Philosophical Logic, vol. 14 (1985), pp. 297349. Zbl 0588.03002. MR 87d:03023. 231

[2] Yablo, S., "Paradox without self-reference," Analysis, vol. 53 (1993), pp. 251-52. Zbl 0943.03565. MR 94i:03008. 231

[3] Yanofsky, N. S., "A universal approach to self-referential paradoxes, incompleteness and fixed points," The Bulletin of Symbolic Logic, vol. 9 (2003), pp. 362-86. MR 2004h:03116. 231 


\section{Acknowledgments}

Thanks to Professor Noson Yanofsky for critically reading and commenting on preliminary versions of this paper. Thanks also to Ms. Margaret D'Souza for typing and proofchecking this paper.

Tata Institute of Fundamental Research

School of Technology \& Computer Science

Mumbai 400005

INDIA

raja@tifr.res.in

http://www.tcs.tifr.res.in/ raja 